\title{
Corrigendum
}

\section{Host immunity contributes to the anti-melanoma activity of BRAF inhibitors}

Deborah A. Knight, Shin Foong Ngiow, Ming Li, Tiffany Parmenter, Stephen Mok, Ashley Cass, Nicole M. Haynes, Kathryn Kinross, Hideo Yagita, Richard C. Koya, Thomas G. Graeber, Antoni Ribas, Grant A. McArthur, and Mark J. Smyth

Original citation: J Clin Invest. 2013;123(3):1371-1381. doi:10.1172/JCI66236.

Citation for this corrigendum: J Clin Invest. 2016;126(1):402-403. doi:10.1172/JCI84828.

An error was made during the preparation of Figure 6B, resulting in more lines on the graph than the number of samples shown. In addition, the legend for Figure 6 did not indicate that WT plus vehicle and WT plus PLX4720 samples shown in Figure 6B are also presented in Figure 6C. All samples in these panels were derived from the same set of experiments. The authors have revisited the statistical analyses to account for the number of comparisons appropriately. The corrected Figure 6 and sentences for the legend are below.

Figure 6

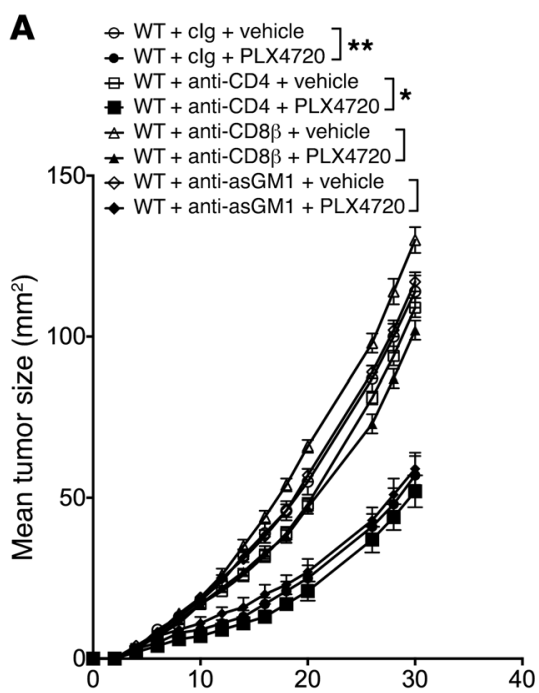

Days after SM1WT1 tumor inoculation

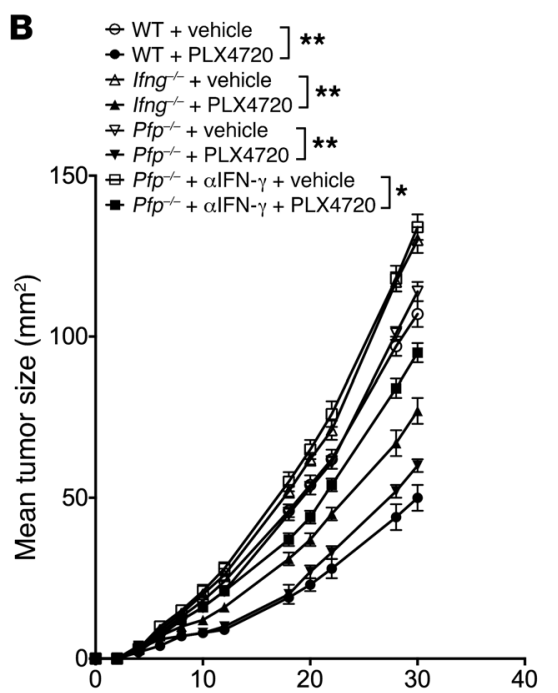

Days after SM1WT1 tumor inoculation

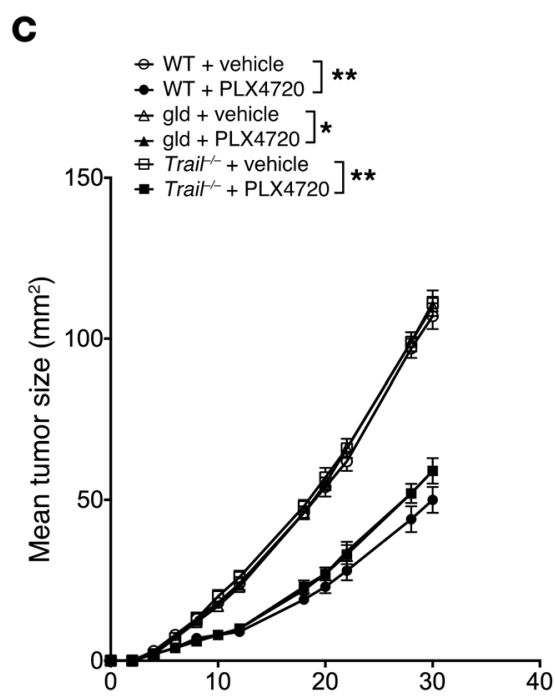

Days after SM1WT1 tumor inoculation

Statistical differences in tumor sizes between mice treated with vehicle and those treated with PLX4720 therapy for each group were determined by a Kruskal-Wallis test with Dunn's correction $\left({ }^{\star} P<0.05 ;{ }^{* *} P<0.01\right)$. WT plus vehicle and WT plus PLX4720 samples shown in $\mathbf{B}$ are also presented in $\mathbf{C}$. All samples in panels $\mathbf{B}$ and $\mathbf{C}$ were derived from the same set of experiments.

Several panels in Supplemental Figure 4 for SM1 staining were accidentally duplicated. The original raw data were reanalyzed and replotted and are shown in the updated version of the supplemental data file. The PD-L1 panel for PLX4720 $10 \mu \mathrm{M}$ was omitted in the corrected version, as there appeared to be a processing error with the original sample. The authors have stated that subsequent replicate experiments are consistent with these findings.

The legend for Supplemental Figure 9A did not indicate that the WT plus vehicle and WT plus anti-CD137 plus vehicle groups shown are presented again in Supplemental Figure 9B. The online supplemental data file has been updated to correct this issue, and the statistical analysis has been revisited to account for the number of comparisons appropriately. None of the corrections alter the conclusions of the paper. 
The legend for Figure 9 did not indicate that the WT plus cIg plus vehicle and WT plus cIg plus PLX4720 groups shown are also presented in Supplemental Figure 7A. All samples in these panels were derived from the same set of experiments. The authors revisited the statistical analyses to account for the number of comparisons appropriately. Supplemental Figure 7 has been updated in the online supplemental data file. The corrected Figure 9 and sentences for the legend are shown below.

Figure 9

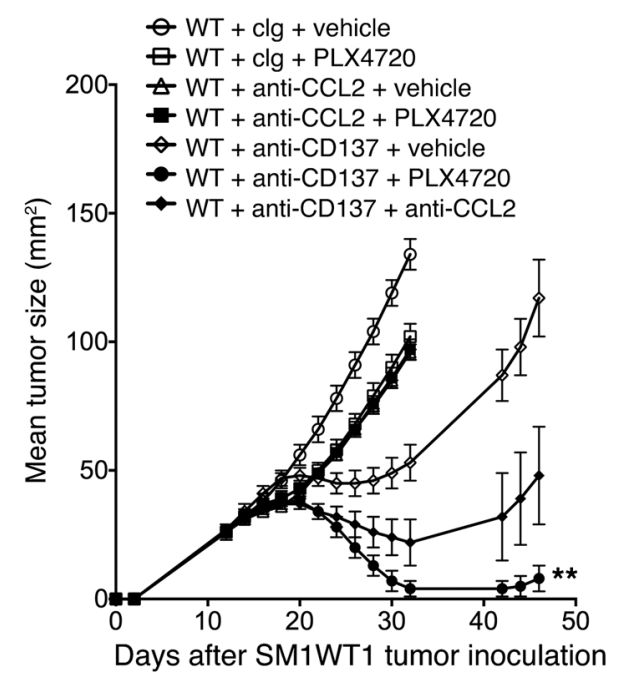

Statistical differences in tumor sizes between mice treated with anti-CD137 and those treated with combination therapy for each group were determined by a Kruskal-Wallis test with Dunn's correction $\left({ }^{* *} P<0.01\right)$. The WT plus cIg plus vehicle and WT plus cIg plus PLX4720 groups shown are also presented in Supplemental Figure 7A. All samples in Figure 9 and Supplemental Figure 7A were derived from the same set of experiments.

The authors originally presented the same data in different panels for the sake of figure clarity and regret the subsequent confusion. The authors regret the other figure errors.

\section{Corrigendum}

\section{Identification and validation of $\mathrm{N}$-acetyltransferase 2 as an insulin sensitivity gene}

Joshua W. Knowles, Weijia Xie, Zhongyang Zhang, Indumathi Chennamsetty, Themistocles L. Assimes, Jussi Paananen, Ola Hansson, James Pankow, Mark O. Goodarzi, Ivan Carcamo-Orive, Andrew P. Morris, Yii-Der I. Chen, Ville-Petteri Mäkinen, Andrea Ganna, Anubha Mahajan, Xiuqing Guo, Fahim Abbasi, Danielle M. Greenawalt, Pek Lum, Cliona Molony, Lars Lind, Cecilia Lindgren, Leslie J. Raffel, Philip S. Tsao, The RISC (Relationship between Insulin Sensitivity and Cardiovascular Disease) Consortium, The EUGENE (European Network on Functional Genomics of Type Diabetes) Study, The GUARDIAN (Genetics UndeRlying DIAbetes in HispaNics) Consortium, The SAPPHIRe (Stanford Asian and Pacific Program for Hypertension and Insulin Resistance) Study, Eric E. Schadt, Jerome I. Rotter, Alan Sinaiko, Gerald Reaven, Xia Yang, Chao A. Hsiung, Leif Groop, Heather J. Cordell, Markku Laakso, Ke Hao, Erik Ingelsson, Timothy M. Frayling, Michael N. Weedon, Mark Walker, and Thomas Quertermous

Original citation: J Clin Invest. 2015;125(4):1739-1751. doi:10.1172/JCI74692.

Citation for this corrigendum: J Clin Invest. 2016;126(1):403. doi:10.1172/JCI85921.

Indumathi Chennamsetty's name was spelled incorrectly in the author list. The correct author list is above.

The authors regret the error. 